\title{
Ueber die Endlichkeit der Invarianten binärer Formen
}

\author{
R. Weitzenbock (Amsterdam).
}

Communicated by H. W. TuRnBdLl.

(Received 24th November 1932. Read 20th January 1933.)

In der Theorie der Invarianten binärer Formen gilt der Satz, dass die projektiven Komitanten eines vollen Systems durch sukzessives Ueberschieben berechnet werden können. Hierauf beruht der Originalbeweis für die Endlichkeit, den P. Gordan 1868 zuerst gegeben hat. ${ }^{1}$ Konstruktiv wird dieser Beweis aber erst dann, wenn man von vorneherein eine obere Grenze für den Grad der Komitanten eines vollen Systems in den Koeffizienten der Grundformen angeben kann. Also eine natürliche Zahl $N$, die die Sicherheit gibt, dass alle Komitanten eines vollen Systems gefunden sind, wenn man die Ueberschiebungen soweit berechnet hat, dass ihr Grad $N$ nicht übersteigt.

Der Originalbeweis Gordans eignet sich nicht für die Bestimmung einer solchen Schranke. Dagegen ergibt sich aus einem, durch Mertens $^{2}$ gegebenen Endlichkeitsbeweis in natürlicher Weise ein Verfahren, das zur Ermittlung von $N$ führt; wir folgen deshalb in unserer Darstellung dem Beweise von Mertens.

Die gefundene Schranke ist nur von theoretischem Werte, da sie viel zu hoch ist; aber es handelt sich ja im Prinzipe nur um die tatsächliche Aufweisung einer endlichen natürlichen Zahl für den allgemeinen Fall. In speziellen Fällen wird sich meist eine viel kleinere Schranke ermitteln lassen.

Da sich die Ermittlung der.Invarianten von $\mathbf{n}$-ären Formen bezüglich $r$-gliedriger linearer Gruppen auf die Berechnung der projektiven Invarianten binärer Formen zurückführen lässt, ist es auch für die erstgenannten Komitanten möglich eine obere Gradschranke anzugeben, worauf ich an anderer Stelle näher eingehen werde.

1 P. Gordan, Journal für Math., 69 (1868), 323-354; vgl. auch: GordnkKersch ensteiner, Vorles. iiber Invariantentheorie 2, Leipzig (1887), 231.

2 F. Mertens, Journal für Math., 100 (1887), 223-230, Vgl. auch D. Hilbert, Math. Annalen, 33 (1889), 223-226 ; E. B. Elliot, Algebra of Quantics, Oxford (1895), 188 ; Grace and Young, The Algebra of Invariants, Cambridge (1903), 101. 
$\S 1$.

Es sei $\{F\}$ ein System von binären Grundformen, das nicht aus einer einzigen Linearform besteht und das die Form $f_{n}=a_{x}^{n}(n \geqq 1)$ enthält.

$$
K(F)=A_{1}, A_{2}, \ldots ; B_{1}(x), B_{2}(x), \ldots
$$

sei ein kleinstes volles System von ganzen, rationalen, projektiven Komitanten von $\{F\}: A_{\nu}$ seien die Invarianten, $B_{\nu}(x)$ die Kovarianten von $K(F)$.

Wir nennen $N(F)$ die Anzahl der Komitanten (1), $N_{c}(F)$ den Höchstgrad dieser Komitanten in allen Formenkoeffizienten zusammen und $N_{x}(F)$ den Höchstgrad in $x_{1} / x_{2}$ bei den Kovarianten $B_{\nu}(x)$. Es ist dann $N(F) \geqq 2$ und $N_{x}(F) \geqq n$.

Wir nehmen zu $\{F\}$ die Linearform

$$
P=(p x)=p_{1} x_{2}-p_{2} x_{1}
$$

als weitere Grundform hinzu. Hierdurch entsteht das Grundformensystem $\{F, P\}$ mit dem vollen System

$$
K(F, P)=A_{1}, A_{2}, \ldots ; P ; Q_{1}, Q_{2}, \ldots,
$$

wobei die Komitanten $Q_{i}$ aus den $B_{\nu}(x)$ durch Polarisieren mit der Reihe $p_{1} / p_{2}$ erhalten werden. Ist also z.B. $B_{\nu}(x)=\alpha_{x}^{\sigma}$, dann sind

$$
a_{x}^{\sigma}, \alpha_{x}^{\sigma-1} \alpha_{p}, a_{x}^{\sigma-2} a_{p}^{2}, \ldots, \alpha_{p}^{\sigma}
$$

unter den $Q_{i}$ von (3) zu finden.

Für die zum vollen System $K(F, P)$ gehörigen Maximalzahlen finden wir dann nach (4), da aus jedem $B_{\nu}(x)$ vom Grade $\sigma$ in den $x_{i}$ genau $\sigma$ neue Komitanten $Q_{i}$ entstehen, $\sigma \leqq N_{x}(F)$ und die Anzahl der Kovarianten $B_{\nu}(x)$ kleiner als $N(F)$ ist:

$$
N(F, P)=N(F)+1+\sum_{B} \sigma<N(F)+1+N(F) \cdot N_{x}(F)
$$

oder, wenn wir kurz $N, N_{x}$ und $N_{c}$ für $N(F), N_{x}(F)$ und $N_{c}(F)$ schreiben:

$$
\begin{aligned}
& N(F, P)<N .\left(1+N_{x}\right) \\
& N_{x}(F, P)=N_{x} .
\end{aligned}
$$

Bei der Bestimmung von $N_{c}(F, P)$ sind die $p_{i}$ als Formenkoeffizienten zu betrachten; da die $Q_{i}(x)$ höchstens vom Grade $N_{x}$ in den $p_{i}$ werden, haben wir

$$
N_{c}(F, P) \leqq N_{x}+N_{c} .
$$

Wir betrachten jetzt alle Komitanten $G$ von $\{F, P\}$, die in den $p_{i}$ und in den Koeffizienten $a_{i k l \ldots}$ von $f_{n}=a_{x}^{n}$ von gleichem Grade ( $\geqq 0$ ) 
sind und beweisen nach Mertens, dass alle diese $G$ einen endlichen Integritätsbereich bilden.

Wir verteilen die in (3) stehenden Polynome von $K(F, P)$ in drei Klassen $S_{i}$ :

(6) $S_{1}=P_{1}, P_{2}, \ldots, P_{v} ; \quad S_{2}=P_{\nu+1}, P_{v+2}, \ldots, P_{\rho}$ und $S_{3}=R_{1}, R_{2}, \ldots, R_{\sigma}$. $\mathrm{Zu} S_{1}$ rechnen wir diejenigen $\nu$ Basiskomitanten $P_{i}$ von $K(F, P)$, bei denen die Differenz: Grad in den Koeffizienten von $\{F\}$ vermindert um den Grad in $p_{i}$, eine positive ganze Zahl $a_{i}>0$ ist; zu $S_{2}$ rechnen wir diejenigen, bei denen diese Differenz gleich Null und zu $S_{3}$ schliesslich die, bei denen diese Differenz $=-b_{\kappa}<0$ wird. Für die Zahlen $\nu$ und $\sigma$ haben wir dann neben $\nu<\rho$ :

$$
N(F) \leqq \nu<N(F, P)-1 \text { und } 1 \leqq \sigma<N(F, P)-N\left(F^{\prime}\right)
$$

also nach (5a):

$$
N \leqq \nu<N .\left(N_{x}+1\right) \text { und } 1 \leqq \sigma<N . N_{x} .
$$

Jede Komitante $G$ aus $K(F, P)$ zerfällt jetzt nach (6) in Glieder der Gestalt

$$
G=P_{1}^{a_{1}} P_{2}^{a_{2}} \ldots P_{\nu}^{a_{\nu}} \ldots P_{\rho}^{\alpha_{\rho}} R_{1}^{\beta_{1}} R_{2}^{\beta_{2}} \ldots R_{\sigma \sigma}^{\beta^{\beta}}
$$

wobei die Exponenten einer Gleichung genügen:

$$
a_{1} a_{1}+a_{2} \alpha_{2}+\ldots+a_{\nu} a_{\nu}=b_{1} \beta_{1}+b_{2} \beta_{2}+\ldots+b_{\sigma} \beta_{\sigma} .
$$

Hier betrachten wir die ganzen, nicht-negativen Zahlen $a_{i}$ und $b_{k}$ als gegebene Koeffizienten, die $\alpha_{r}$ und $\beta_{s}$ hingegen als ganzzahlige Unbekannte $\geqq 0$. (8) hat dann eine endliche Lösungsbasis und deren $\tau$ Basislösungen erhält man nach MERTENs wie folgt. Ist $t_{i k}$ der grösste gemeinsame Teiler von $a_{i}$ und $b_{k}$, dann bilden

$$
\alpha_{i}=\frac{b_{\kappa}}{t_{i \kappa}}, \quad \beta=\frac{a_{i}}{t_{i \kappa}},
$$

alle anderen $a_{r}$ und $\beta_{s}$ gleich Null eine Basislösung von (8) und es gibt $\tau_{1}=\nu \sigma$ solche Lösungen. Neben diesen kann es noch $\tau_{2}$ weitere Basislösungen geben, bei denen die linke Seite von (8) höchstens gleich der Zahl ist.

$$
s=\sum_{i=1}^{\nu} \sum_{\kappa=1}^{\sigma} \frac{a_{i} b}{t_{i \kappa}}-\nu \sigma
$$

Wegen

$$
\begin{aligned}
s & \leqq\left(\sum_{i=1}^{\nu} a_{i}\right) \cdot\left(\sum_{\kappa=1}^{\sigma} b_{\kappa}\right)-\nu \sigma \leqq \nu\left(a_{i}\right)_{\max .} \cdot \sigma\left(b_{\kappa}\right)_{\max .}-\nu \sigma \\
& \leqq \nu \sigma\left[N_{c}(F) \cdot N_{x}(F)-1\right]
\end{aligned}
$$

wird nach (7): 


$$
s<N^{2} N_{x}\left(N_{x}+1\right)\left(N_{c} N_{x}-1\right)=S .
$$

Es gilt also für diese $\tau_{2}$ weiteren Basislösungen :

$$
a_{1} a_{1}+a_{2} a_{2}+\ldots+a_{v} a_{v}<S
$$

hieraus folgt wegen $a_{i} \geqq 1$ :

$$
a_{i}<S, \quad \beta_{\kappa}<S
$$

und also $\tau_{2}<$ als die grössere der Zahlen $S^{\nu}, S^{\sigma}$, somit wieder nach (7):

$$
\tau_{2}<S^{N(N}{ }^{+1)} \text {. }
$$

Daher ergibt sich für die Anzahl $\tau_{1}+\tau_{2}$ der Basislösungen.

$$
\tau=\tau_{1}+\tau_{2}<\nu \sigma+S^{N\left(N_{x}+1\right)}
$$

d. h. nach (7) und (11):

$$
\tau<N^{2} N_{x}\left(N_{x}+1\right)+\left[N^{2} N_{x}\left(N_{x}+1\right)\left(N_{c} N_{x}-1\right)\right]^{N\left(N_{x}+1\right)} .
$$

Die mit diesen $\tau$ Basislösungen gebildeten Komitanten

$$
G=P_{1}^{a_{1}} P_{2}^{a_{2}} \ldots P_{\nu}^{a_{\nu}} R_{1}^{\beta_{1}} R_{2}^{\beta_{2}} \ldots R_{\sigma}^{\beta_{\sigma}}
$$

bilden mit den $\rho-\nu$ Komitanten $P_{\nu+1}, \ldots, P_{\rho}$ von (6) zusammen eine Integritätsbasis $G_{1}, G_{2}, \ldots, G_{\gamma}$ für alle $G$. Da $\rho \leqq N(F, P)-1$ ist, folgt nach (5a):

$$
\rho-\nu \leqq N(F, P)-2 \leqq N(N+1)-1 .
$$

Für die Anzahl $\gamma$ der Basiskomitanten $G_{i}$ ergibt sich also:

$$
\gamma=\tau+\rho-\nu
$$

$<N\left(N_{x}+1\right)-1+N^{2} N_{x}\left(N_{x}+1\right)+\left[N^{2} N_{x}\left(N_{x}+1\right)\left(N_{c} N_{x}-1\right)\right]^{N\left(N_{x}+1\right)}$.

Für die Gradzahlen der Komitanten (6) gelten (5b) und (5c). Nach (13) haben wir daher für den Grad $\gamma_{x}$ von $G_{i}$ in den $x_{1} / x_{2}$ :

$$
\begin{aligned}
\gamma_{x} & \leqq\left(\sum_{i=1}^{\nu} \alpha_{i}+\sum_{\kappa=1}^{\sigma} \beta_{\kappa}\right) \cdot N_{x}(F, P) \\
& <(\nu+\sigma) \cdot S \cdot N_{x},
\end{aligned}
$$

also nach (7) und (11):

$$
\gamma_{x}<N^{3} N_{x}^{2}\left(2 N_{x}+1\right)\left(N_{x}+1\right)\left(N_{x} N_{c}-1\right) .
$$

Auf dieselbe Weise erhalten wir für den Grad $\gamma_{c}$ der Basiskomitanten $A_{i}$ in den Koeffizienten der Formen $\{F, P\}$ :

$$
\begin{aligned}
\gamma_{c} & \leqq\left(\sum_{i=1}^{\nu} \alpha_{i}+\sum_{\kappa=1}^{\sigma} \beta_{\kappa}\right) \cdot N_{c}(F, P) \\
& <(\nu+\sigma) \cdot S \cdot\left(N_{x}+N_{c}\right) \\
\gamma_{c} & <N^{3} N_{x}\left(N_{x}+1\right)\left(2 N_{x}+1\right)\left(N_{x}+N_{c}\right)\left(N_{x} N_{c}-1\right) .
\end{aligned}
$$




\section{$\S 2$.}

Es sei nun $\left\{F^{\prime}\right\}$ das System binärer Grundformen, das aus $\{F\}$ entsteht, wenn man die Form $f_{n}=a_{. r}^{n}$ ersetzt durch eine Form $(n+1)$-ten Grades $f_{n+1}=A_{x}^{n+1}$ und die übrigen Grundformen ungeändert lässt. Ist dann $H$ eine Komitante des Systems $\left\{F^{\prime}\right\}$, in der die Koeffizienten von $f_{n+1}$ wirklich auftreten, und ersetzt man diese Koeffizienten von $f_{n+1}$ durch die entsprechenden des Produktes $f_{n} . P$, so entsteht aus $H$ eine Komitante $G^{\prime \prime}$ von $\{F, P\}$, die in den Koeffizienten von $f_{n}$ und in den $p_{1} / p_{2}$ vom selben Grade ist. $G^{\prime \prime}$ ist daher durch die $G_{1}, G_{2}, \ldots, G_{\gamma}$ des vorigen Abschnitt ganz und rational ausdrückbar, d. h. wird eine Summe von Potenzprodukten

$$
\Theta=G_{1}^{\mu_{1}} G_{2}^{\mu_{2}} \ldots G_{\gamma}^{\mu}
$$

Jetzt führen wir $n$ Linearformen

$$
Q=q_{1} x_{2}-q_{2} x_{1}, \quad R=r_{1} x_{2}-r_{2} x_{1}, \ldots, S=s_{1} x_{2}-s_{2} x_{1}
$$

ein und ersetzen in jedem $G_{i}$ die Koeffizienten von $f_{n}$ durch die entsprechenden der Produktform $Q . R \ldots S$. Hierdurch entstehen aus den $G_{i}$ Formen $G_{i 1}$, die symmetrisch in den $n$ Reihen $q_{i}, r_{i}, \ldots, s_{i}$ sind; aus $\Theta$ entsteht ein $\Theta_{1}$ und aus $G^{\prime \prime}$ entsteht eine Form

$$
G_{1}^{\prime \prime}=\Sigma G_{11}^{\mu_{1}} G_{21}^{\mu_{2}} \ldots G_{\gamma{ }^{1}}^{\mu_{\gamma}}
$$

die symmetrisch ist in allen $n+1$ Reihen $p, q, r, \ldots, s$. Hieraus folgt, dass wir eindeutig von $G_{1}{ }^{\prime \prime}$ zum ursprünglichen $H$ zurückgelangen, wenn wir in $G_{1}^{\prime \prime}$ jedes Produkt $p_{i} q_{k} r_{l} \ldots$ durch den Koeffizienten $A_{i k l \ldots}$. von $f_{n+1}$ ersetzen.

In (19) vertauschen wir zuerst die $p_{i}$ mit den $q_{i}$; dies gibt

dann die $p_{i}$ mit den $r_{i}$ :

$$
G_{1}^{\prime \prime}=\Sigma G_{12}^{\mu_{1}} G_{22}^{\mu_{2}} \ldots G_{\gamma 2}^{\mu}
$$

$$
G_{1}^{\prime \prime}=\Sigma G_{13}^{\mu_{1}} G_{23}^{\mu_{2}} \ldots G_{\gamma 3}^{\mu},
$$

u.s.f.; zum Schlusse die $p_{i}$ mit den $s_{i}$. Alles addiert gibt:

$$
(n+1) . G_{1}^{\prime \prime}=\Sigma G_{11}^{\mu_{1}} \ldots G_{\gamma 1}^{\mu}+\Sigma G_{12}^{\mu_{1}} \ldots G_{\gamma 2}^{\mu_{\gamma}^{-}}+\ldots+\Sigma G_{1, n+1}^{\mu_{1}} \ldots G_{\gamma_{, n+1}}^{\mu}
$$

Gehen wir jetzt aus von

$$
\begin{aligned}
S_{m}=\left(u_{1} G_{11}+\ldots+u_{\gamma} G_{\gamma 1}\right)^{m} & +\left(u_{1} G_{12}+\ldots+u_{\gamma} G_{\gamma 2}\right)^{m} \\
& +\ldots+\left(u_{1} G_{1, n+1}+\ldots u_{\gamma} G_{\gamma, n+1}\right)^{m}
\end{aligned}
$$

dann ist in $S_{m}$ der Koeffizient von $w_{1}^{\mu_{1}} u_{z}^{\mu_{2}} \ldots u_{\gamma}^{\mu}$ bis auf einen Zahlenfaktor gleich $\operatorname{dem} G_{1}{ }^{\prime \prime}$ von (20) mit $\mu_{1}+\mu_{2}+\ldots+\mu_{\gamma}=m$. Nun ist 
jedes $S_{m}$ ganz und rational durch diejenigen Polynome $G_{1}{ }^{\prime \prime}$ auszudrücken, für welche

$$
\mu_{1}+\mu_{2}+\ldots+\mu_{\gamma} \leqq n+1
$$

wird. Wir haben also als Integritätsbasis aller $G_{1}{ }^{\prime \prime}$ eine endliche Anzahl $\pi$ von Polynomen

$$
G_{1, \alpha}^{\prime \prime}=\sum_{i} G_{1 i}^{a_{1}} G_{: i}^{\alpha_{2}} \ldots G_{\gamma^{i}}^{a_{\gamma}} \quad\left(\alpha_{1}+a_{2}+\ldots+\alpha_{\gamma} \leqq n+1\right)
$$

und wenn wir in diesen $p_{i} q_{k} r_{l} \ldots=A_{i k l \ldots}$ setzen, erhalten wir eine Integritätsbasis für alle Komitanten $H$. Die Anzahl $\pi$ dieser Basiskomitanten $H$ ist dann höchstens gleich der Zahl der Koeffizienten aller Potenzprodukte $u_{1}^{a_{1}} u_{2}^{a_{2}} \ldots \ldots u_{\gamma}^{a_{\gamma}}$ in $S_{1}, S_{2}, \ldots, S_{n+1}$, also, da $S_{m}$ genau $\left(\begin{array}{c}\gamma+m-1 \\ m\end{array}\right)$ Koeffizienten enthält:

$$
\pi \leqq\left(\begin{array}{l}
\gamma \\
1
\end{array}\right)+\left(\begin{array}{c}
\gamma+1 \\
2
\end{array}\right)+\ldots+\left(\begin{array}{c}
\gamma+n \\
n+1
\end{array}\right)=\left(\begin{array}{c}
\gamma+n+1 \\
n+1
\end{array}\right) .
$$

Somit gilt für die Anzahl der Basiskomitanten von $\left\{F^{\prime}\right\}$ :

$$
N\left(F^{\prime}\right) \leqq\left(\begin{array}{c}
\gamma+n+1 \\
n+1
\end{array}\right)+N(F, P)
$$

wobei für $\gamma$ die Ungleichung (14) und für $N(F, P)$ die Ungleichung (5a) gilt.

Weiters haben wir für den Grad $\gamma^{\prime \prime}{ }_{x} \operatorname{der}$ Komitanten $G^{\prime \prime}{ }_{1, a}$ von (23):

$$
\gamma^{\prime \prime}{ }_{x} \leqq\left(a_{1}+a_{2}+\ldots+a_{y}\right) \cdot \gamma_{x} \leqq(n+1) \gamma_{x} .
$$

Daher ist $N_{x}\left(F^{\prime}\right)$ höchstens gleich der grösseren der Zahlen $(n+1) \gamma_{x}$ und $N_{x}(F, P)=N_{x}$, also nach (15):

$$
N_{x}\left(F^{\prime}\right)<(n+1) N^{3} N_{x}\left(2 N_{x}+1\right)\left(N_{x}+1\right)\left(N_{x} N_{c}-1\right) .
$$

Auf dieselbe Art erhält man nach (16):

$$
N_{c}\left(F^{\prime}\right)<(n+1) N^{3} N_{x}\left(2 N_{x}+1\right)\left(N_{x}+1\right)\left(N_{x}+N_{c}\right)\left(N_{x} N_{c}-1\right) .
$$

Hiemit haben wir für $N\left(F^{\prime}\right), N_{x}\left(F^{\prime}\right)$ und $N_{c}\left(F^{\prime}\right)$ obere Schranken gefunden, die nur von $n$ und den Zahlen $N(F), N_{x}(F)$ und $N_{c}\left(F^{\prime}\right)$ des Grundformensystems $\left\{F^{\prime}\right\}$ abhängen. Ist $n \geqq 2$, dann kann man die letzten drei Zahlen wieder abschätzen, ausgehend von einem Grundformensystem $\left\{F^{(-1)}\right\}$, das aus $\left\{F^{\prime}\right\}$ entsteht, wenn $f_{n}$ durch $f_{n-1}$ ersetzt wird u.s.f. Schliesslich gelangt man zu einem System $\{L\}$ von binären Linearformen $L_{1}, L_{2}, \ldots, L_{j}(j \geqq 2)$ und für dieses gilt:

$$
N(L)=j+\frac{1}{2} j(j-1), \quad N_{x}(L)=1, \quad N_{c}(L)=\mathbf{2} .
$$


$\S 3$.

Um einfachere Ausdrücke zu erhalten, vergrössern wir jetzt die gefundenen Schranken nach oben. Nennen wir $M$ die grösste der drei Zahlen $N(F), N_{x}(F)$ und $N_{c}(F)$ und schliessen den Fall aus, dass $\{F\}$ aus einer einzigen binären qudratischen Form besteht, so können wir $n+1 \leqq M$ und $M \geqq 3$ voraussetzen.

Nach (14) haben wir dann

$$
\gamma<M(M+1)+M^{3}(M+1)+\left[M^{3}(M+1) M^{2}\right]^{M(M+1)} ;
$$

da $M+1 \leqq \frac{4}{3} M$ und $M^{2}+1 \leqq \frac{10}{9} M^{2}$ ist, wird dies zu

also

$$
\begin{aligned}
\gamma & <\frac{4}{3} M^{2}+\frac{4}{3} M^{4}+\left(\frac{4}{3} M^{6}\right)^{3 M^{2}} \\
& <\frac{40}{27} M^{4}+M^{5 M^{2}}\left(\frac{4}{3}\right)^{\frac{3}{M^{2}}}<M^{5}+M^{8 M^{2}+2 M^{2}} \\
& <2 M^{Z_{3}^{3} M^{2}}=M^{2 \xi^{3} M^{2}+\log 2 / \log M},
\end{aligned}
$$

(28)

$$
\gamma<M^{9 M^{2}}
$$

Nach (24) und (5a) ergibt sich

$$
\begin{aligned}
N\left(F^{\prime}\right) & \leqq \frac{\gamma+n+1}{n+1} \cdot \frac{\gamma+n}{n} \ldots \frac{\gamma+1}{1}+M(M+1) \\
& <(\gamma+1)^{n+1}+\frac{4}{3} M^{2} \\
& <\left(1+M^{9 M^{2}}\right)^{M}+\frac{4}{3} M^{2} \\
& <2\left(2 M^{9 M^{2}}\right)^{M}, \\
\text { (29) } \quad N\left(F^{\prime}\right) & <M^{10 M^{3}} .
\end{aligned}
$$

In ähnlicher Weise folgen aus (25) und (26):

$$
N_{x}\left(F^{\prime}\right)<\frac{32}{9} M^{11} \text { und } N_{c}\left(F^{\prime}\right)<\frac{64}{9} M^{10} .
$$

Schreiben wir jetzt $M_{n}$ statt $M$ und nennen $M_{n+1}$ die grösste der drei Zahlen $N\left(F^{\prime}\right), N_{x}\left(F^{\prime}\right)$ und $N_{c}\left(F^{\prime}\right)$, so folgt:

Führen wir eine Funktion $\phi$ ein durch

$$
\phi(a)=\phi_{1}(a)=a^{10 a^{2}}
$$

und setzen

$$
\phi_{2}(a)=\phi(\phi(a)), \quad \phi_{3}(a)=\phi\left(\phi_{2}(a)\right), \ldots,
$$

so können wir nach (31) schreiben:

$$
M_{n}<\phi_{1}\left(M_{n-1}\right)<\phi_{2}\left(M_{n-2}\right)<\ldots<\phi_{k}\left(M_{n-k}\right) .
$$


Sei nun ein System $\{F\}$ von $s \geqq 1$ Grundformen

$$
f_{n_{1}}, f_{n_{2}}, \ldots, f_{n_{1}}
$$

der Grade $n_{1}, n_{2}, \ldots, n_{8}$ in $x_{1} / x_{2}$ gegeben und wenigstens ein $n_{i} \geqq 2$. Dann ist

$$
M(F)<\phi\left(M\left(F_{1}\right)\right),
$$

wo $\left\{F_{1}\right\}$ aus $\{F\}$ entsteht, wenn $f_{n_{i}}$ durch eine Grundform $f_{n_{i}-1}$ ersetzt wird. Ebenso ist

$$
M\left(F_{1}\right)<\phi\left(M\left(F_{2}\right)\right)
$$

wo $\left\{F_{2}\right\}$ aus $\left\{F_{1}\right\}$ entsteht, indem man entweder $f_{n_{i}-1}$ durch eine $f_{n_{i}-2}$ oder eine $f_{n_{k}}$ durch eine $f_{n_{k}-1}$ ersetzt. Setzen wir also

$$
d=n_{1}+n_{2}+\ldots+n_{s}-s,
$$

so sind wir für $s>1$ nach $d$ Schritten bei einem System $\{L\}$ von Linearformen angelangt, wofür also

$$
M(L)=s+\left(\begin{array}{l}
s \\
2
\end{array}\right)<s^{2}
$$

ist. Wenn aber $s=1$ und $n_{1} \geqq 2$ ist, so sind wir nach $d-1=n_{1}-2$ Schritten bei einer binären quadratischen Form mit $M=2$ angelangt. In allen diesen Fällen ist

$$
M(F)<\phi_{d}\left(s^{2}+1\right) .
$$

Lassen wir jetzt auch den Fall zu, dass $\{F\}$ nur Linearformen enthält, dann ist $d+1=1$ und

$$
M(F)<\phi_{d+1}\left(s^{2}+1\right),
$$

d. h. wir haben in allen Fällen

$$
M(F)<\phi_{d+1}\left(s^{2}+1\right) .
$$

Dies gibt den Satz:

Ist $f_{n_{1}}, f_{n_{y}}, \ldots, f_{n_{s}}$ ein System von $s$ binären Grundformen der Grade $n_{1}, n_{2}, \ldots, n_{s}$, und ist $K_{1}, K_{2}, \ldots, K_{m}$ ein volles System von projektiven Komitanten dieser Grundformen, so ist die Anzahl $m$ dieser $K_{i}$, der Grad eines $K_{i}$ in den $x_{1}: x_{2}$ und der Grad eines $K_{i}$ in den Koeffizienten der Grundformen

$$
<\phi_{d+1}\left(s^{2}+1\right) \text {, }
$$

wo $d=n_{1}+n_{2}+\ldots+n_{s}-s$ ist und $\phi_{d+1}(a)$ die $(d+1)$-fach iterierte Funktion

bedeutet.

$$
\phi(a)=a^{10 a^{3}}
$$

